Progress Report

for Period May 1, 1992 -December 31, 1992

\section{T. Alan Hatton}

Department of Chemical Engineering Massachusetts Institute of Technology Cambridge MA 02139

December 1992

Prepared for

THE U.S. DEPARTMENT OF ENERGY AGREEMENT NO. DE-FG02-92-ER14262 


\section{NOTICE}

This report was prepared as an account of work sponsored by the United States Government. Neither the United States nor the Department of Energy, nor any of their employees, nor any of their contractors, subcontractors, or their employees, makes any warranty, express or implied, or assumes any legal liability or responsibility for the accuracy, completeness, or usefulness of any information, apparatus, product or process disclosed or represents that its use would not infringe privately-owned rights. 


\begin{abstract}
The solubilization of a range of polycyclic aromatic hydrocarbons in block copolymer micelles has been studied as a function of polymer composition, architecture, and temperature. Micelle formation is favored at high temperatures, leading to significant enhancements in solubilization capacity. At low temperatures, however, micelles do not form and the solubilization capacity of the block copolymer solution for the organics is low; this provides a convenient method for the regeneration of micellar solutions used as "solvents" in the treatment of contaminated feed streams using membrane extraction principles. It has also been shown (in collaboration with K.P. Johnston of University of Texas, Austin) that supercritical $\mathrm{CO}_{2}$ can be used effectively for micelle regeneration.

Theoretical calculations of the structure of block copolymer micelles in the presence and absence of solutes using self-consistent mean-field lattice theories have successfully captured the trends observed with changing polymer composition and architecture, often quantitatively. The temperature and composition dependence of the micellar properties were determined by allowing the individual polymer segments to assume both polar and non-polar conformations.
\end{abstract}




\section{Introdurtion}

Micelles formed by polypropylene oxide (PPO) and polyethylene oxide (PEO) block copolymers can strongly solubilize certain polycyclic aromatic hydrocarbons. We have suggested that this may be exploited in the treatment of contaminated aqueous streams by using block copolymer solutions retained within the lumens of hollow fibre membrane modules to extract contaminants from aqueous feed streams fed to the shell side of the module. The basic concept has been demonstrated, but information is still needed on how the micellar solutions can be regenerated so that the polymers can be recycled for further use. The progress made over the last six months on this project has been primarily in investigating the effect of temperature on the solubilising capacity of block copolymer solutions. It is suggested here that the temperature dependence of solubilization can be utilized to both maximize solubilization and regenerate the polymer solution. Additional work on the regeneration of the micellar solution using supercritical $\mathrm{CO}_{2}$, done in collaboration with Johnston at the University of Texas, Austin, is also discussed briefly.

\section{Theoretical Analysis}

The self-consistent mean-field lattice theory for the modelling of block copolymer micelles in the presence and absence of solute was used successfully to interpret observed experimental trends based on polymer composition and architecture, particuarly once we accounted for the polar and apolar conformations available to individual polymer segments. Two papers were prepared and submitted based on this work, which was completed under DOE funding; these papers are currently undergoing revision in response to some minor suggestions of the reviewers. Preprints are attached.

\section{Experimental Procedure}

Saturated micellar solutions of polycyclic aromatic hydrocarbons were prepared using the generating column technique in which the polymer solution was pumped continuously through a generating column packed with solute crystals or flakes. A small stream of the polymer solution was diverted to a continuous flow spectrophotometer cell after passing through the generating column. Flow cells with path lengths of $0.01 \mathrm{~mm}$ and $0.1 \mathrm{~mm}$ were used to maintain the level of absorbance below unity. The absorbance was measured on a Lambda 3B UV/VIS Spectrophotometer at the wavelength of maximum absorbance for pyrene, $272 \mathrm{~nm}$.

The generating column and the reservoir containing the polymer solution were immersed in a constant temperature water bath, monitored by a $\mathrm{CH} / \mathrm{P}$ temperature control system circulator. To maintain accurate temperature control throughout the system, tubing leading to and from the pump and to the spectrophotometer was insulated. The temperature of the polymer solution was recorded using a thermometer in the solution reservoir.

Once equilibrium was reached at the desired temperature, a small sample of the polymer solution was taken and analyzed on a Waters 410 Differential Refractometer to determine polymer concentration. The refractometer was connected to a Shimadzu CR3A integrator which measured both the area and height of the peak. The temperature control system was then adjusted to raise the temperature of the water bath and the process was repeated for a select temperature range for each of the polymer solutions. At the conclusion of each experiment, temperature was decreased to its initial value. When equilibrium was reached the pyrene and polymer concentrations were measured. Three block copolymers were tested at three different concentrations.

With benzene or toluene, saturated aqueous solutions in equilibrium with the bulk organic liquids 
were cloudy, owing to the formation of large microemulsion drops stabilized by the polymeric surfactants; some of the polymer was also lost to the organic phase. In an attempt to overcome this limitation, head-space gas chromatogrophy was used to estimate the partition coefficient between the micelle core and the water phase as a function of the solute concentration. This work was done in collaboration with Peter Carr of the Chemistry Department at the University of Minnesota.

\section{Results and Discussion}

The polymers used in this study are listed in Table 1.

The enhanced solubility of pyrene in P104 with changes in temperature is shown in Figure 1. At low temperatures, there was effectively no enhancement in solubility. Above some transition temperature, there was a rapid increase in enhancement over a short temperature range, followed by a third regime in which the increase in solubility became less significant. This second transition temperature increased with decreasing polymer concentration, being $28.2,27.5$ and $24.1{ }^{\circ} \mathrm{C}$ for the $1 \%, 4 \%$ and $8 \%$ solutions, respectively. The increase in solubility at higher temperatures was predominantly a result of an increase in the water solubility of pyrene. Figure 2 shows the solubility of pyrene in the polymer solution normalized by its solubility in water. Above the transition temperature the normalized concentration of pyrene was independent of temperature, which indicates a constant partition coefficient for the solute between the micelle core and the bulk water.

The solubilizing behavior of the P104 solution as temperature increases can be attributed to the enhanced terndency of the polymer to aggregate and form micelles, which grow in size as water becomes a poorer solvent for PPO and PEO. Below the first transition temperature, the polymer has not yet become sufficiently hydrophobic for micelle growth to occur, and thus the solubilisation of pyrene is not enhanced. Above the second transition temperature, the micelle size (aggregation number) remains constant,and provides an effectively unchanging environment for the solute, leading to a constant partition coefficient in this regime. The strong changes observed in solubility enhancement in the intermediate region are a result of the rapid growth of the micelles in this region.

Similar behavior was observed for aqueous solutions of F108 and L64. For F108, regions of micellar growth and constant micellar size were observed for both 4 and $7 \%$ solutions, the transition temperature decreasing from 34.3 to $31.7^{\circ} \mathrm{C}$ with the increase in polymer concentration. A transition temperature was not observed for the $1 \%$ solution of F108 within the range of the experiments; it is possibly above $40^{\circ} \mathrm{C}$. The normalized pyrene solubility curve showed micellar saturation for both the $4 \%$ and $7 \%$ solutions above their transition temperatures. A uniform increase in solubilization of pyrene with temperature for L64 was observed, which suggests that this copolymer exhibits only the two regions of no micellar formation and micellar growth, respectively, no transition from micellar growth to constant micelle size being observed for any concentration in the temperature range of the experiments. The micelles formed with L64 never showed a region in which the pyrene partition coefficient was constant at any of the concentrations studied.

Figure 3 compares the solubility of pyrene in $8 \%$ solutions of P104, F108 and L64 at various temperatures. The P104 solution demonstrated the greatest solubilization of pyrene with a maximum $C / C_{\text {sat }}$ of approximately 6600 . The maximum solubility of pyrene in F108 was significantly lower at 1200 , while the L64 micelles did not reach a maximum level of pyrene solubilization. P104 both has a higher molecular weight and is more hydrophobic than either L64 and F108, and therefore solubilized the most pyrene. Partition coefficients were calculated for the 
three polymers and are reported in table 2 , where

$$
\mathrm{K}_{\mathrm{mw}}=\mathrm{cs}_{\mathrm{m}} / \mathrm{cs} \mathrm{w}_{\mathrm{w}}
$$

and $c^{s}$ and $c_{w}$ are the concentrations of pyrene in the micelle (based on total polymer mass) and in the water, respectively. The $\mathrm{K}_{\mathrm{mw}}$ was determined from the slope of the plot of pyrene solubility vs. polymer concentration. The $\mathrm{K}_{\mathrm{mw}}$ at $400^{\circ} \mathrm{C}$ for $\mathrm{L} 64$ was appioximately half that of P104, while the $\mathrm{K}_{\mathrm{mw}}$ for $\mathrm{F} 108$ was lower by a factor of almost 5 .

The effect of solute on partitioning can be explored by a comparison to the results of the naphthalene partitioning experiments of $\mathrm{Hurter}^{2}$. Solubilization was more greatly enhanced for pyrene than for naphthalene. The maximum pyrene solubility $\left(C / C_{s a t}\right)$ was 6600 for a $8 \%$ solution of P104, while Hurter ${ }^{2}$ measured a maximum naphthalene solubility enhancement of 200 in a $10 \%$ P104 solution. This increased solubilization was expected since the two additional benzene rings in pyrene cause it to have a lower aqueous solubility and higher hydrophobicity than naphthalene. Hurter2 established

$$
\log \mathrm{K}_{\mathrm{mw}} \quad \alpha \log \mathrm{K}_{\mathrm{ow}}
$$

where $\mathrm{K}_{\mathrm{ow}}$ is the octanol/water partition coefficient for the solute and is generally used as a measure of hydrophobicity. The results from the pyrene temperature experiments are consistent with this relationship. Although solubility enhancement is greater, the solute load in the micelle ( $\mathrm{g}$ solute/g polymer) is less for pyrene. The micelle load was $0.015 \mathrm{~g}$ pyrene/g P104 and $0.1 \mathrm{~g}$ naphthalene/g P104.

The L64 solubility curve has a lower position relative to the P104 and F108 curves for pyrene than naphthalene, indicating that L64 has a lower potential for solubilizing pyrene than naphthalene relative to P104 and F108. This difference is most likely due to the low molecular weight of L64. Since pyrene is a larger molecule than naphthalene, the smaller hydrophobic core of L64 may hinder the solubilization of pyrene over naphthalene (the PPO molecular weight for L64 is half that of P104 and F108).

In collaboration with Professor Peter Carr of the Chemistry Department at the University of Minnesota, we conducted some preliminary experiments using head-space gas chromatography to estimate the solubility characteristics of benzene in block copolymer solutions. The results were not totally reliable owing to experimental complications associated with the microsyringe pumps used to feed the benzene to the polymer solution. Nonetheless, it was evident that, with more time, this approach could be valuable for the study of the solubilisation characteristics of volatile organics. The partition coefficients for benzene were in accord with expectations based on extrapolation of the earlier results on naphthalene, phenanthrene and pyrene using the octano;/water hydrophobicity scale.

\section{Solution Regeneration}

For all polyrners and concentrations, a precipitate was observed upon cooling the solution and the maximurn absorbance corresponded with that measured as temperature was increased. The polymer concentration was also consistent with that measured throughout each experiment. These results indicate that the polymer can be regenerated by cooling the solution to a temperature at which micelle formation was not observed. At this temperature, pyrene solubility is low, causing 
the additionai pyrene to precipitate out of solution. Furthermore, the solid solute can be easily removed so that the polymer solution can be reused.Not all block copolymers will exhibit this behaviour, however; some may be able to be regenerated by increases in temperature, while if polyampholytic polymers are used, changes in $\mathrm{pH}$ may be effective for the recovery and recycle of the polymers.

The loaded micellar solution can be contacted with a low molecular weight solvent having a high capacity for the solutes to remove the contaminating species. Any residual solvent left with the micellar phase can then be flashed off before the micelles are recycled. In a collaboration with Johnston at the University of Texas, we have shown that supercritical $\mathrm{CO}_{2}$ can be used effectively for micellar regeneration. Shown in Figure 4 are the extraction efficiencies for a single stage as a function of pressure. Approximately $85-90 \%$ of the naphthalene was removed in this operation, which is sufficient for the regeneration and recycling of the solvent phase.In practical applications it may be advantageous to first ultrafilter the micellar solution to remove much of the suspending water to reduce the volume of aqueous micellar solution to be regenerated.

\section{Conclusions}

The solubilization of pyrene by poly(ethylene oxide-propylene oxide) block copolymer micelles is enhanced with increasing temperature. A greater hydrophobicity of the polymer solution contributes to the increased partitioning of pyrene to the micelles. High molecular weight and high PPO:PEO ratio both result in high hydrophobicity, with an increase in \% PPO content having a greater effect on enhancing solubilization than an increase in molecular weight. As the concentration of the polymer solution increased, the temperature for the cessation of micellar growth decreased, a manifestation of the temperature effect on the critical micelle concentration.

The regeneration of the block copolymer micellar solutions was shown to be possible using either temperature swings or supercritical $\mathrm{CO}_{2}$ extraction. The economic effectiveness of these strategies stills needs to be addressed.

Molecular modelling of the micelle-forming block copolymer solutions and their propensity to enhance the solubility of trace organics using self-consistent field theories was found to be very successful. In many cases semi-quantitative predictions of partitioning results were obtained.

\section{Reforences}

(1) Hurter, P.N., Hatton, T.A., Langmuir. 1992, 8, 1291-1299.

(2) Hurter, P.N., Solubilization of Polycyclic Aromatic Hydrocarbons in Block Copolymer Micelles: Partitioning Experiments and Molecular Modelling ; Massachusetts Institute of Technology, Department of Chemical Engineering: Cambridge, MA 02139.

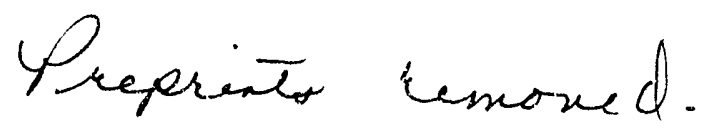


Table 1. Physical Properties of the Polymers

\begin{tabular}{lccc} 
& $\begin{array}{c}\text { Molecular } \\
\text { Weight }\end{array}$ & $\begin{array}{c}\text { PPO } \\
\text { Content (\%) }\end{array}$ & $\begin{array}{r}\text { PPO Molecular } \\
\text { Weight }\end{array}$ \\
\hline P104 & 5900 & 60.0 & 3072 \\
L64 & 2900 & 60.0 & 1510 \\
F108 & 14600 & 20.0 & 3091
\end{tabular}

Table 2. Partition Coefficients measured at $400 \mathrm{C}$ $\mathrm{K}_{\mathrm{mw}}$

\begin{tabular}{ll}
\hline P104 & 81000 \\
L64 & 49000 \\
F108 & 18000
\end{tabular}




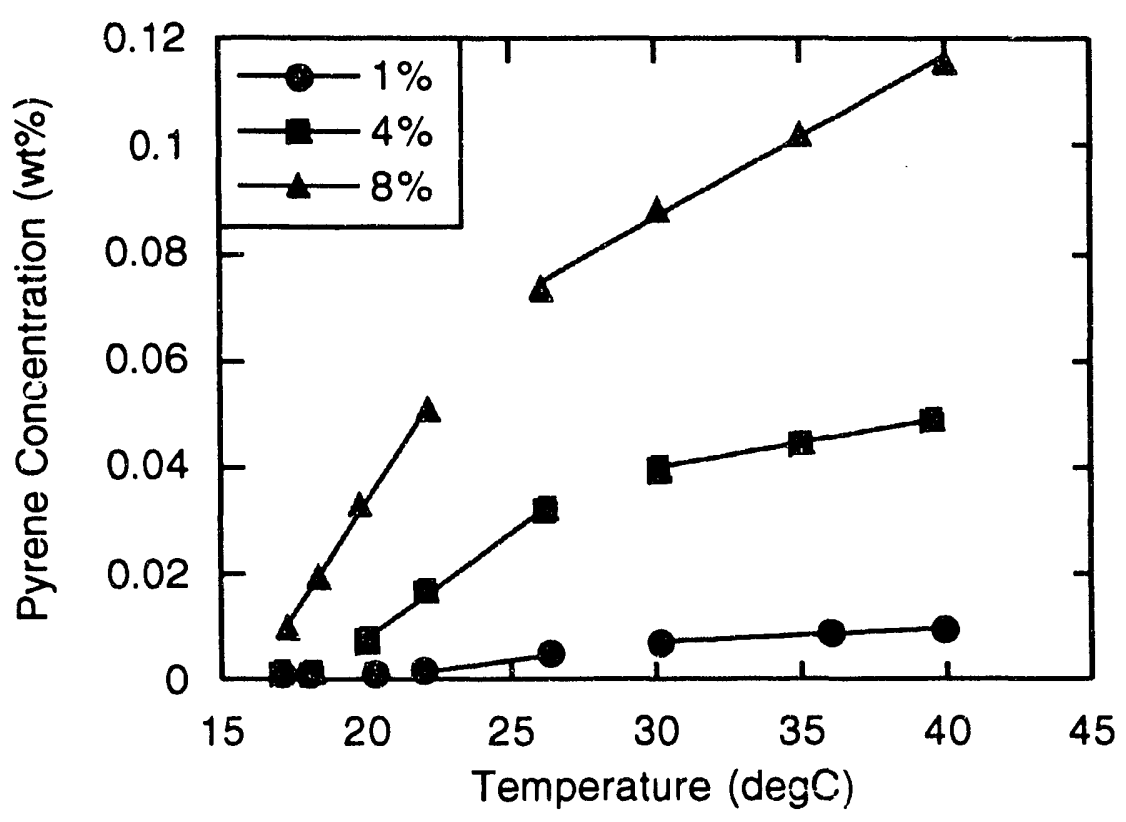

Figure 1. Effect of temperature and P104 concentration on the solubilisation of pyrene.

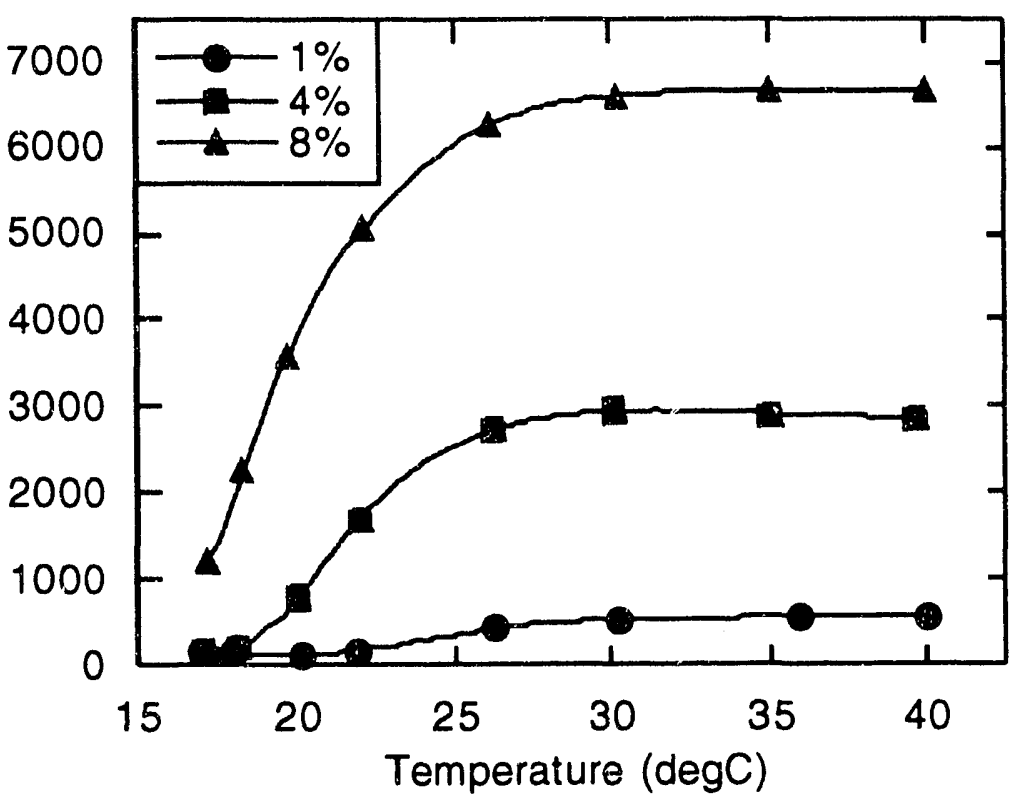

Figure 2 Relative Solubility of pyrene in solutions of P104 as a function of temperature. 


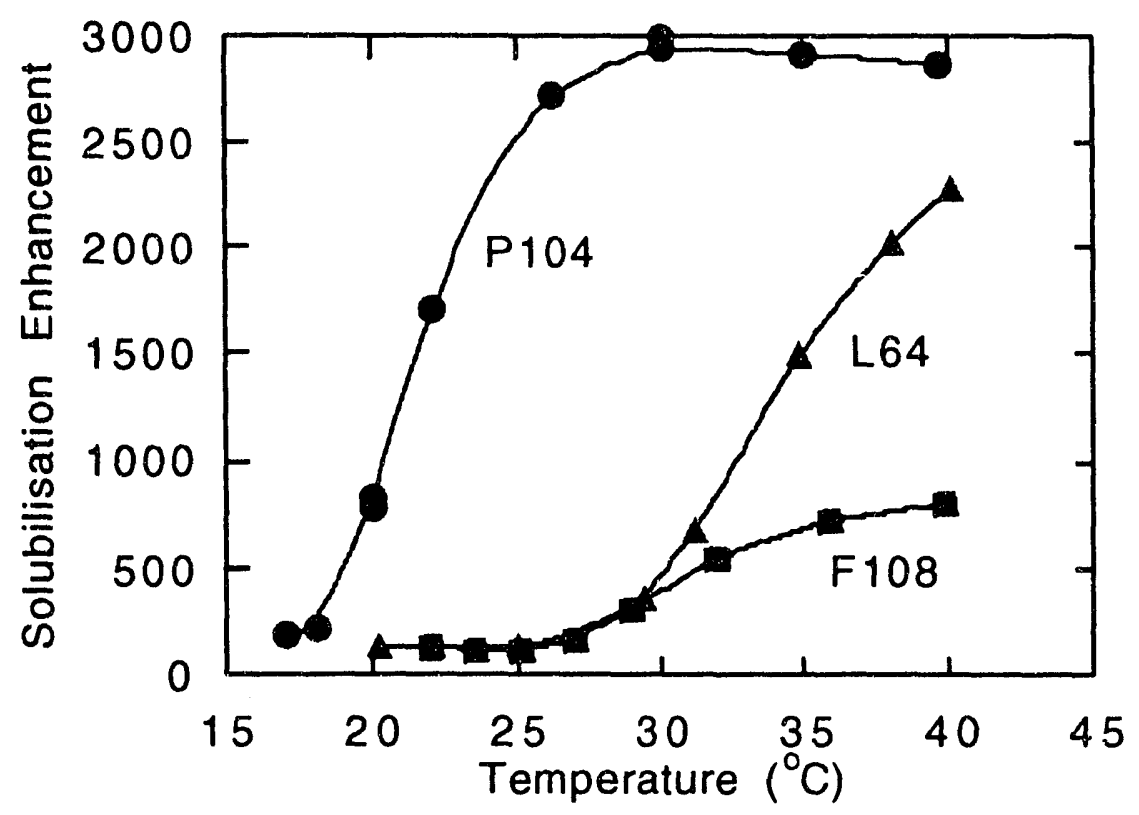

Figure 3 Enhanced solubilisation of pyrene in solutions of block copolymers of different molecular weights and PPO content

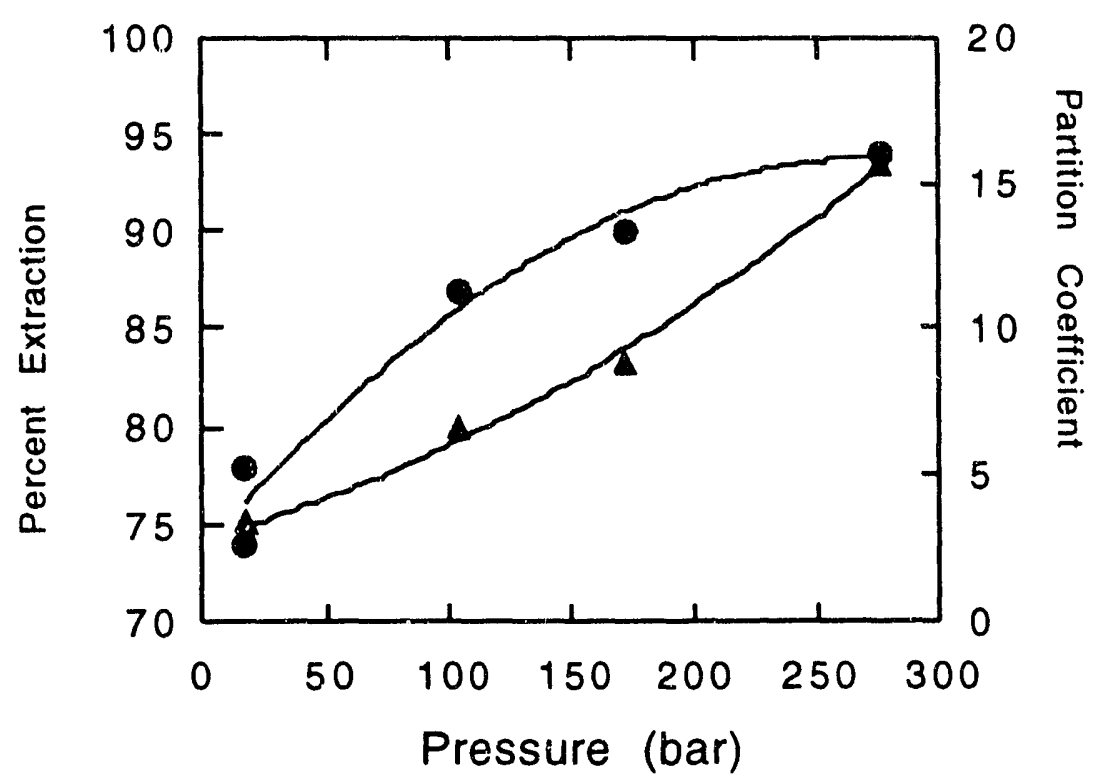

Figure 4. The percent extraction of pyrene from P104 micelles in a single stage extraction using supercritical carbon dioxide. 
